\title{
On the algebraic Riccati equation
}

\section{Harald K. Wimmer}

In this note the matrix equation $A+W B+B^{T} W+W C W=0$ is considered. A monotoneity result and an inertia theorem on the location of the eigenvalues of $W$ and $B+C W$ are proved.

\section{A monotoneity result}

We study the algebraic Riccati equation

$$
A+W B+B^{T} W+W C W=0,
$$

where all matrices are $n \times n$ and real, and $A, C$, and $W$ are symmetric. We assume

$$
C \leq 0 \quad \text { (negative semidefinite) }
$$

and

$$
(B, C) \text { controllable. }
$$

[See the next section for the definition of controllability.] Coppel [4] has given a comprehensive algebraic theory of (I) on which we base our note.

We recall the following results from [4]. If (1) is solvable, then there exists a maximal solution $W_{+}$; that is, $W_{+} \geq \tilde{W}$ for each solution $\tilde{W}$ of (1). If $M$,

$$
M=\left(\begin{array}{cc}
B & C \\
-A & -B^{T}
\end{array}\right),
$$

has no eigenvalues on the imaginary axis, then there exists a solution of

Received 11 March 1976. This research was supported by the Alexander von Humboldt-Stiftung. 
(1) and $B+C W$ is a stable matrix (that is with all eigenvalues in the left half plane), if and only if $W=W_{+}$. Set

$$
H=\left(\begin{array}{cc}
A & B^{T} \\
B & C
\end{array}\right)
$$

Then (1) can be written as

$$
(I \quad W) H\left(\begin{array}{l}
I \\
W
\end{array}\right)=0
$$

THEOREM 1. Let $M$ have no eigenvalues on the imaginamy axis and let $W_{+}$be the maximal solution of (1). If $W_{1}$ is a solution of

$$
A_{1}+W_{1} B_{1}+B_{1}^{T} W_{1}+W_{1} C_{1} W_{1}=0
$$

and

$$
H=\left(\begin{array}{cc}
A & B^{T} \\
B & C
\end{array}\right) \geq H_{1}=\left(\begin{array}{ll}
A_{1} & B_{1}^{T} \\
B_{1} & C_{1}
\end{array}\right)
$$

then $w_{+} \geq w_{1}$.

Proof. $H_{1}$ can be written as $H_{1}=H-\tilde{H}, \tilde{H} \geq 0$. Thus (4) is equivalent to

$$
A+W_{1} B+B^{T} W_{1}+W_{1} C W_{1}=\left(I W_{1}\right) \tilde{H}\left(\begin{array}{c}
I \\
W_{1}
\end{array}\right)=R \geq 0 .
$$

For $D=W_{+}-W_{1}$ we obtain

$$
D\left(B+C W_{+}\right)+\left(B+C W_{+}\right)^{T} D=D C D-R
$$

which is a Ljapunov matrix equation with $B+\mathrm{CW}_{+}$stable and $D C D-R \leq 0$. Hence $D \geq 0$. This theorem generalizes a result in [7] where monotoneity with respect to $A$ was proved.

\section{An inertia theorem}

We shall need the following lemmas and definitions. The pair $(F, G)$, $F \in C^{n \times n}, G \in C^{n \times m}$, is called controllable (Hautus [5]), if 


$$
\operatorname{rank}\left(G, F G, \ldots, F^{n-1} G\right)=n
$$

The pair $\left(\begin{array}{l}F \\ K\end{array}\right), K \in C^{p \times n}$, is called observable [2], if $\left(F^{T}, K^{T}\right)$ is controllable.

LEMMA 1 (Hautus [5]). The pair $\left(\begin{array}{l}F \\ K\end{array}\right)$ is observable, if and only if for $\lambda \in C, y \in C^{n}$,

$$
F y=\lambda y, \quad K y=0 \Rightarrow y=0 .
$$

For a complex $n \times n$ matrix $F$ the inertia, in $F$, of $F$ is defined [6] as the triple

$$
\text { in } F=\{\pi(F), \nu(F), \delta(F)\}
$$

where $\pi(F), \nu(F)$, and $\delta(F)$ are respectively the number of eigenvalues of $F$ with positive, negative, and vanishing real part.

LEMMA 2 ([3], [8]). Let $F, W$, and $S$ be real $n \times n$ matrices, $S$ and $W$ symmetric. If $S \leq 0$ and $\left(\begin{array}{l}F \\ S\end{array}\right)$ is observable and

$$
W F+F^{T} W=S
$$

then

(a) in $F=$ in $(-W)$ and $\delta(F)=\delta(W)=0$, and especially

(b) $F$ is stable, if and only if $W>0$.

The following inertia result is obtained under additional assumptions on the coefficient matrices of ( 1 ).

THEOREM 2. Assume $C \leq 0,(B, C)$ controlzable,

$$
A \geq 0
$$

and

$$
\left(\begin{array}{l}
B \\
A
\end{array}\right) \text { observabie. }
$$

Then

(a) there exists a solution of (1), and 
(b) for each solution W,

$$
\operatorname{in}(B+C W)=\operatorname{in}(-W) \text { and } \delta(B+C W)=\delta(W)=0
$$

holds.

Proof. (a) We show that $\delta(M)=0$, which implies the existence of a solution. Suppose $i \alpha, \alpha$ real, is an eigenvalue of $M$ with eigenvector $\left(\begin{array}{l}m \\ s\end{array}\right)$; that is,

$$
M\left(\begin{array}{l}
2 \\
s
\end{array}\right)=i \alpha\left(\begin{array}{l}
r \\
s
\end{array}\right),\left(\begin{array}{l}
m \\
s
\end{array}\right) \neq\left(\begin{array}{l}
0 \\
0
\end{array}\right)
$$

Premultiplying both sides of (8) by $\left(s^{*}, r^{*}\right)$, we get

$$
s^{*} C s-r^{*} A r+\left(s^{*} B r-r^{*} B^{T} s\right)=i \alpha\left(s^{*} r^{+}+r^{*} s\right),
$$

and, separating real and imaginary parts, we obtain

$$
s^{*} C s-r^{*} A r=0 \text {. }
$$

(2) and (6) imply $C s=0$ and $A r=0$. Now (8) yields $B r=i a r$ and $B^{T} s=-i \alpha s$. Thus $\left(\begin{array}{c}B-i \alpha I \\ A\end{array}\right) r=0$, and since $\left(\begin{array}{l}B \\ A\end{array}\right)$ is assumed to be observable, we deduce from Lemma 1 that $r=0$. Similarly $s=0$. Therefore $i \alpha$ can not be an eigenvalue of $M$. A different proof that there exists a solution $W$ relies on optimal control theory and can be found in [2].

(b) Let $W$ be a solution of (1). Then

$$
W(B+C W)+(B+C W)^{T} W=W C W-A .
$$

From $(W C W-A) q=0$ we get $C W q=0$ and $A q=0$. Therefore $(B+C W) q=\lambda q$ and $(W C W-A) q=0$ implies

$$
B q=\lambda q, A q=0 .
$$

Because of (7) and (9) we have $q=0$ and the pair $\left(\begin{array}{c}B+C W \\ W C W-A\end{array}\right)$ is also observable. The conditions of Lemma 2 are satisfied and the statement of the inertia follows.

One of the problems which lead to (1) with coefficients satisfying (3), (4), (6), and (7) is the output regulator problem over an infinite time interval (see [1]). This close connection between optimal control 
theory and the algebraic Riccati equation has been used to establish the following result (see [2]), for which an algebraic proof is now immediate.

THEOREM 3 ([2]). Let (1) be given together with (3), (4), (6), and (7). Then $W_{+}$is the only positive definite solution of (1).

Proof. Since $B+C W_{+}$is stable, $W_{+}>0$ follows from Theorem 2 (b). Conversely, if $W>0$, then by the same theorem $A+C W$ is stable, which is only possible for $W=W_{+}$.

\section{References}

[1] Michael Athans, Peter L. Falb, Optimal control. An introduction to the theory and its applications (McGraw-Hill, New York, St. Louis, San Francisco, Toronto, London, Sydney, 1966).

[2] Roger W. Brockett, Finite dimensional linear systems (John Wiley \& Sons, New York, London, Sydney, Toronto, 1970).

[3] Chi-Tsong Chen, "A generalization of the inertia theorem", SIAM J. App 2. Math. 25 (1973), 158-161.

[4] W.A. Coppel, "Matrix quadratic equations", Bull. Austral. Math. Soc. $10(1974), 377-401$.

[5] M.L.J. Hautus, "Controllability and observability conditions of linear autonomous systems", Proc. K. Nederl. Akad. Wetensch. Amsterdam Ser. A 72 (1969), 443-448.

[6] Alexander Ostrowski and Hans Schneider, "Some theorems on the inertia of general matrices", J. Math. Anal. Appl. 4 (1962), 72-84.

[7] Jan C. Willems, "Least squares stationary optimal control and the algebraic Riccati equation", IEEE Trans. Automatic Control AC-16 (1971), 621-634.

[8] Harald K. Wimmer, "Inertia theorems for matrices, controllability, and linear vibrations", Linear AZgebra and Appl. 8 (1974), 337-343.

Mathematisches Institut der Universität, Würzburg, Germany;
Mathematisches Institut, Technische Universität Graz, Graz, Austria. 